\title{
Polarizations as States and Their Evolution in Geometric Algebra Terms with Variable Complex Plane
}

\author{
Alexander Soiguine \\ SOiGUINE Quantum Computing, Aliso Viejo, CA, USA \\ Email: alex@soiguine.com
}

How to cite this paper: Soiguine, A. (2018) Polarizations as States and Their Evolution in Geometric Algebra Terms with Variable Complex Plane. Journal of Applied Mathematics and Physics, 6, 704-714. https://doi.org/10.4236/jamp.2018.64063

Received: March 7, 2018

Accepted: April 17, 2018

Published: April 20, 2018

Copyright $\odot 2018$ by author and Scientific Research Publishing Inc. This work is licensed under the Creative Commons Attribution International License (CC BY 4.0).

http://creativecommons.org/licenses/by/4.0/

\section{c) (i) Open Access}

\begin{abstract}
Recently suggested scheme [1] of quantum computing uses g-qubit states as circular polarizations from the solution of Maxwell equations in terms of geometric algebra, along with clear definition of a complex plane as bivector in three dimensions. Here all the details of receiving the solution, and its polarization transformations are analyzed. The results can particularly be applied to the problems of quantum computing and quantum cryptography. The suggested formalism replaces conventional quantum mechanics states as objects constructed in complex vector Hilbert space framework by geometrically feasible framework of multivectors.
\end{abstract}

\section{Keywords}

Quantum Mechanics, Quantum Computing, Geometric Algebra, Maxwell Equations

\section{Introduction}

The circular polarized electromagnetic waves are the only type of waves following from the solution of Maxwell equations in free space done in geometric algebra terms.

Let's take the electromagnetic field in the form:

$$
F=F_{0} \exp \left[I_{S}(\omega t-\boldsymbol{k} \cdot \boldsymbol{r})\right]
$$

requiring that it satisfies the Maxwell system of equations in free space, which in geometrical algebra terms is one equation:

$$
\left(\partial_{t}+\nabla\right) F=0
$$


where $\nabla=\frac{\partial}{\partial x} \hat{x}+\frac{\partial}{\partial y} \hat{y}+\frac{\partial}{\partial z} \hat{z}$ and multiplications are the geometrical algebra ones.

Element $F_{0}$ in (1) is a constant element of geometric algebra $G_{3}$ and $I_{S}$ is unit value bivector of a plane $S$ in three dimensions, that is a generalization of the imaginary unit [2], [3]. The exponent in (1) is unit value element of $G_{3}^{+}$[3]:

$$
\mathrm{e}^{I_{S} \varphi}=\cos \varphi+I_{S} \sin \varphi, \varphi=\omega t-\boldsymbol{k} \cdot \boldsymbol{r}
$$

Solution of (2) should be sum of a vector (electric field $E$ ) and bivector (magnetic field $\left.I_{3} \boldsymbol{H}\right)$ :

$$
F=\boldsymbol{E}+I_{3} \boldsymbol{H}
$$

with some initial conditions:

$$
\boldsymbol{E}+\left.I_{3} \boldsymbol{H}\right|_{t=0, r=0}=F_{0}=\left.\boldsymbol{E}\right|_{t=0, r=0}+\left.I_{3} \boldsymbol{H}\right|_{t=0, r=0}=\boldsymbol{E}_{0}+I_{3} \boldsymbol{H}_{0}
$$

In the magnetic field $I_{3} \boldsymbol{H}$ the item $I_{3}$ is unit pseudoscalar in three dimensions assumed to be the right-hand screw oriented volume, relative to an ordered triple of orthonormal vectors.

Substitution of (1) into the Maxwell's (2) will exactly show us what the solution looks like.

\section{Solution in the Geometric Algebra Terms}

The derivative by time gives

$$
\frac{\partial}{\partial t} F=F_{0} \mathrm{e}^{I_{S} \varphi} I_{S} \frac{\partial}{\partial t}(\omega t-\boldsymbol{k} \cdot \boldsymbol{r})=F_{0} \mathrm{e}^{I_{S} \varphi} I_{S} \omega=F I_{S} \omega
$$

The geometric algebra product $\nabla F$ is:

$$
\nabla F=F_{0} I_{S} \mathrm{e}^{I_{S} \varphi} \nabla(\omega t-\boldsymbol{k} \cdot \boldsymbol{r})=-F_{0} \mathrm{e}^{I_{S} \varphi} I_{S} \boldsymbol{k}=-F I_{S} \boldsymbol{k}
$$

or

$$
\nabla F=F_{0} \mathrm{e}^{I_{S} \varphi} \nabla(\omega t-\boldsymbol{k} \cdot \boldsymbol{r}) I_{S}=-F_{0} \mathrm{e}^{I_{S} \varphi} \boldsymbol{k} I_{S}=-F \boldsymbol{k} I_{S},
$$

depending on do we write $I_{S}(\omega t-\boldsymbol{k} \cdot \boldsymbol{r})$ or $(\omega t-\boldsymbol{k} \cdot \boldsymbol{r}) I_{S}$. The result should be the same since $\omega t-\boldsymbol{k} \cdot \boldsymbol{r}$ is a scalar.

Commutativity $I_{S} \boldsymbol{k}=\boldsymbol{k} I_{S}$ is true only if $\boldsymbol{k} \times I_{3} I_{S}=0$. The following agreement takes place between orientation of $I_{3}$, orientation of $I_{S}$ and direction of vector $\boldsymbol{k}$ [3]. The vector $I_{3} I_{S}=I_{S} I_{3}$ is orthogonal to the plane of $I_{S}$ and its direction is defined by orientations of $I_{3}$ and $I_{S}$. Rotation of right/left hand screw defined by orientation of $I_{S}$ gives movement of right/left hand screw. This is the direction of the vector $I_{3} I_{S}=I_{S} I_{3}$. That means that the matching between $\hat{k}$ and $I_{S}$ should be $\hat{k}= \pm I_{3} I_{S}$ or $\hat{k} I_{S}=\mp I_{3}{ }^{1}$.

Assuming that orientation is $I_{3}=\hat{k} I_{S}$, the Maxwell equation becomes:

$$
F\left(I_{S} \omega-I_{3}|\boldsymbol{k}|\right)=F\left(\omega I_{S}-|\boldsymbol{k}| \hat{k} I_{S}\right)=0
$$

or

${ }^{1}$ For any vector we write $\hat{a}=\boldsymbol{a} /|\boldsymbol{a}|$. 


$$
\left(\boldsymbol{E}+I_{3} \boldsymbol{H}\right) \omega=\left(\boldsymbol{E}+I_{3} \boldsymbol{H}\right) \boldsymbol{k}
$$

Left hand side is sum of vector and bivector, while right hand side is scalar $\boldsymbol{E} \cdot \boldsymbol{k}$ plus bivector $\boldsymbol{E} \wedge \boldsymbol{k}$, plus pseudoscalar $I_{3}(\boldsymbol{H} \cdot \boldsymbol{k})$, plus vector $I_{3}(\boldsymbol{H} \wedge \boldsymbol{k})$. It follows that both $\boldsymbol{E}$ and $H$ lie on the plane of $I_{S}$ and then:

$$
\omega \boldsymbol{E}=I_{3} \boldsymbol{H} \boldsymbol{k}, \omega I_{3} \boldsymbol{H}=\boldsymbol{E} \boldsymbol{k} \rightarrow \frac{\omega^{2}}{|\boldsymbol{k}|^{2}} I_{3} \boldsymbol{H} \boldsymbol{k}=\omega \boldsymbol{E}
$$

Thus, $\omega=|\boldsymbol{k}|$ and we get equation $I_{3} \boldsymbol{H} \hat{k}=\boldsymbol{E}$ from which particularly follows $|\boldsymbol{E}|^{2}=|\boldsymbol{H}|^{2}$ and $\hat{E} \hat{k} \hat{H}=I_{3}$.

The result for the case $I_{3}=\hat{k} I_{S}$ is that the solution of (2) is

$$
F=\left(\boldsymbol{E}_{0}+I_{3} \boldsymbol{H}_{0}\right) \exp \left[I_{S}(\omega t-\boldsymbol{k} \cdot \boldsymbol{r})\right]
$$

where $\boldsymbol{E}_{0}$ and $\boldsymbol{H}_{0}$ are arbitrary mutually orthogonal vectors of equal length, lying on the plane $S$. Vector $\boldsymbol{k}$ should be normal to that plane, $\hat{k}=-I_{3} I_{S}$ and $|\boldsymbol{k}|=\omega$.

In the above result the sense of the $I_{S}$ orientation and the direction of $\vec{k}$ were assumed to agree with $I_{3}=\hat{k} I_{S}$. Opposite orientation, $-I_{3}=\hat{k}_{S}$, that's $\boldsymbol{k}$ and $I_{S}$ compose left hand screw and $\hat{k}=I_{3} I_{S}$, will give solution $F=F_{0} \exp \left[I_{S}(\omega t-\boldsymbol{k} \cdot \boldsymbol{r})\right]$ with $\hat{E} \hat{H} \hat{k}=I_{3}$.

\section{Summary:}

For a plane $S$ in three dimensions Maxwell equation (2) has two solutions

- $F_{+}=\left(\boldsymbol{E}_{0}+I_{3} \boldsymbol{H}_{0}\right) \exp \left[I_{S}\left(\omega t-\boldsymbol{k}_{+} \cdot \boldsymbol{r}\right)\right]$, with $\hat{k}_{+}=I_{3} I_{S}, \hat{E} \hat{H} \hat{k}_{+}=I_{3}$, and the triple $\left\{\hat{E}, \hat{H}, \hat{k}_{+}\right\}$is right hand screw oriented, that's rotation of $\hat{E}$ to $\hat{H}$ by $\pi / 2$ gives movement of right hand screw in the direction of $\quad \boldsymbol{k}_{+}=|\boldsymbol{k}| I_{3} I_{S}$.

- $F_{-}=\left(\boldsymbol{E}_{0}+I_{3} \boldsymbol{H}_{0}\right) \exp \left[I_{S}\left(\omega t-\boldsymbol{k}_{-} \cdot \boldsymbol{r}\right)\right]$, with $\hat{k}_{-}=-I_{3} I_{S}, \hat{E} \hat{H} \hat{k}_{-}=-I_{3}$, and the triple $\left\{\hat{E}, \hat{H}, \hat{k}_{-}\right\}$is left hand screw oriented, that's rotation of $\hat{E}$ to $\hat{H}$ by $\pi / 2$ gives movement of left hand screw in the direction of $\boldsymbol{k}_{-}=-|\boldsymbol{k}| I_{3} I_{S}$ or, equivalently, movement of right hand screw in the opposite direction, $-\boldsymbol{k}_{-}$.

- $E_{0}$ and $H_{0}$, initial values of $E$ and $H$, are arbitrary mutually orthogonal vectors of equal length, lying on the plane $S$. Vectors $\boldsymbol{k}_{ \pm}= \pm\left|\boldsymbol{k}_{ \pm}\right| I_{3} I_{S}$ are normal to that plane. The length of the wave vectors $\left|\boldsymbol{k}_{ \pm}\right|$is equal to angular frequency $\omega$.

Maxwell Equation (2) is a linear one. Then any linear combination of $F_{+}$ and $F_{-}$saving the structure of (1) will also be a solution.

Let's write:

$$
\left\{\begin{array}{l}
F_{+}=\left(\boldsymbol{E}_{0}+I_{3} \boldsymbol{H}_{0}\right) \exp \left[I_{S} \omega t\right] \exp \left[-I_{S}\left[\left(I_{3} I_{S}\right) \cdot \boldsymbol{r}\right]\right] \\
F_{-}=\left(\boldsymbol{E}_{0}+I_{3} \boldsymbol{H}_{0}\right) \exp \left[I_{S} \omega t\right] \exp \left[I_{S}\left[\left(I_{3} I_{S}\right) \cdot \boldsymbol{r}\right]\right]
\end{array}\right.
$$

Then for arbitrary scalars $\lambda$ and $\mu$ :

$$
\lambda F_{+}+\mu F_{-}=\left(\boldsymbol{E}_{0}+I_{3} \boldsymbol{H}_{0}\right) \mathrm{e}^{I_{S} \omega t}\left(\lambda \mathrm{e}^{-I_{S}\left[\left(I_{3} I_{S}\right) \cdot r\right]}+\mu \mathrm{e}^{I_{S}\left[\left(I_{3} I_{S}\right) \cdot r\right]}\right)
$$

is solution of (2). The item in second parenthesis is weighted linear combination 
of two states with the same phase in the same plane but opposite sense of orientation. The states are strictly coupled, entangled if you prefer, because bivector plane should be the same for both, does not matter what happens with it.

One another option is:

$$
\begin{aligned}
& \left(\lambda_{1}+I_{3} \mu_{1}\right)\left(\boldsymbol{E}_{0}+I_{3} \boldsymbol{H}_{0}\right) \exp \left[I_{S} \omega\left(t-\left(I_{3} I_{S}\right) \cdot \boldsymbol{r}\right)\right] \\
& +\left(\lambda_{2}+I_{3} \mu_{2}\right)\left(\boldsymbol{E}_{0}+I_{3} \boldsymbol{H}_{0}\right) \exp \left[I_{S} \omega\left(t+\left(I_{3} I_{S}\right) \cdot \boldsymbol{r}\right)\right] \\
& =\left[\lambda_{1} \boldsymbol{E}_{0}-\mu_{1} \boldsymbol{H}_{0}+I_{3}\left(\mu_{1} \boldsymbol{E}_{0}+\lambda_{1} \boldsymbol{H}_{0}\right)\right] \exp \left[I_{S} \omega\left(t-\left(I_{3} I_{S}\right) \cdot \boldsymbol{r}\right)\right] \\
& +\left[\lambda_{2} \boldsymbol{E}_{0}-\mu_{2} \boldsymbol{H}_{0}+I_{3}\left(\mu_{2} \boldsymbol{E}_{0}+\lambda_{2} \boldsymbol{H}_{0}\right)\right] \exp \left[I_{S} \omega\left(t+\left(I_{3} I_{S}\right) \cdot \boldsymbol{r}\right)\right]
\end{aligned}
$$

which is just rotation, along with possible change of length, of electric and magnetic initial vectors in their plane.

\section{Transformations of Polarization States}

Polarizations, in our approach, exponents in the solution of (3), have the form of states [3], that's elements of $G_{3}^{+}: G_{3}^{+} \ni \alpha+I_{S} \beta \equiv \cos \varphi+I_{S} \sin \varphi=\mathrm{e}^{I_{S} \varphi}$, distributed in $(t, r)$ space. They are operators than can act on observables, also elements of $G_{3}$, particularly other polarizations. Such states can be depicted in the current geometric algebra formalism using a triple of basis bivectors in three dimensions $\left\{B_{1}, B_{2}, B_{3}\right\}$ (Figure 1 ):

The basis bivectors satisfy multiplication rules (in the righth and screw orientation of $I_{3}$ ):

$$
B_{1} B_{2}=-B_{3}, \quad B_{1} B_{3}=B_{2}, \quad B_{2} B_{3}=-B_{1}
$$

One can identify basis bivectors with usual coordinate planes: $B_{1}=\hat{y} \hat{z}$, $B_{2}=\hat{z} \hat{x}, B_{3}=\hat{x} \hat{y}$. Any one of these three bivectors can be taken as explicitly identifying imaginary unit, though any unit value bivector in three dimensions can take the role [2], [4].

Thus:

$$
\alpha+I_{S} \beta=\alpha+\beta\left(b_{1} B_{1}+b_{2} B_{2}+b_{3} B_{3}\right) \equiv \alpha+\beta_{1} B_{1}+\beta_{2} B_{2}+\beta_{3} B_{3}
$$

The difference between units of information in classical computational scheme, quantum mechanical conventional computations (qubits) and geometric algebra
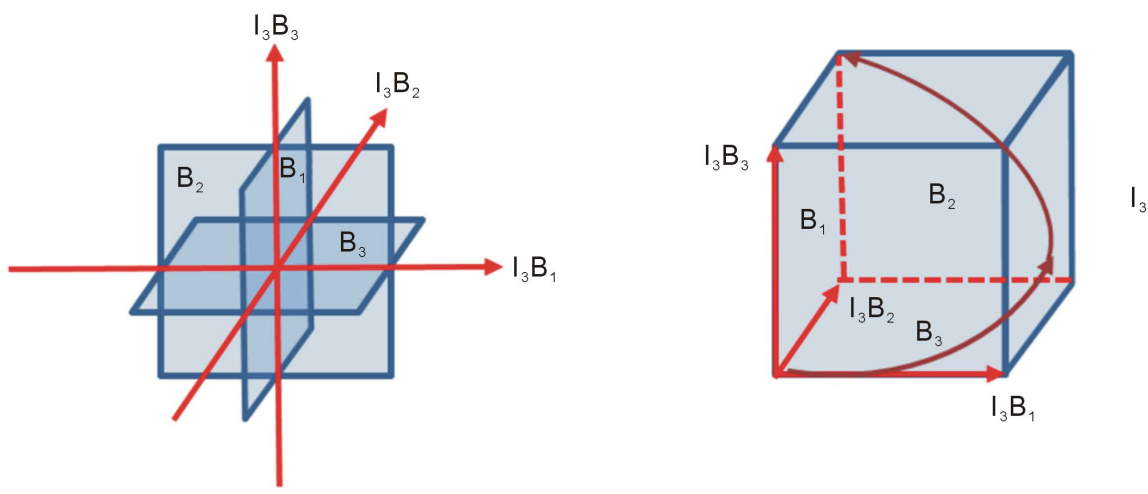

Figure 1. Basis of bivectors and unit value pseudoscalar. 
scheme (g-qubits) with variable explicitly defined complex plane is seen from Figure 2.

Circular polarizations received as solutions of Maxwell Equation (2) is an excellent choice to have such g-qubits in a lab.

Commonly accepted idea to use systems of qubits to tremendously increase speed of computations is based on assumption of entanglement - roughly speaking when touching one qubit all the other in the system react instantly, in no time. A bit strange, though you should not care about that because our paradigm is very different.

Assume we have some general state:

$$
\alpha+I_{S} \beta=\alpha+\beta\left(b_{1} B_{1}+b_{2} B_{2}+b_{3} B_{3}\right) \equiv \alpha+\beta_{1} B_{1}+\beta_{2} B_{2}+\beta_{3} B_{3}
$$

The state can be identified as a point $\left(\alpha_{1}, \beta_{1}, \beta_{2}, \beta_{3}\right)$ on unit sphere $S^{3}$. It can be subjected to a Clifford translation

$$
\alpha+I_{S} \beta \Rightarrow \mathrm{e}^{I_{C I} \Delta \psi}\left(\alpha+I_{S} \beta\right)
$$

executing displacement $\Delta \psi$ at point $\left(\alpha_{1}, \beta_{1}, \beta_{2}, \beta_{3}\right)$ along intersection of $S^{3}$ with the unit bivector plane $I_{C l}$.

Let's make notations more like conventional quantum mechanical ones. I will write:

$$
\alpha+I_{S} \beta \equiv|g\rangle_{\left(\alpha, \beta, I_{S}\right)}, \overline{\alpha+I_{S} \beta}=\alpha-I_{S} \beta \equiv\left\langle\left. g\right|_{\left(\alpha, \beta, I_{S}\right)}\right.
$$

and use Hamiltonian like form of the Clifford translation bivector.

Conventional Hamiltonian
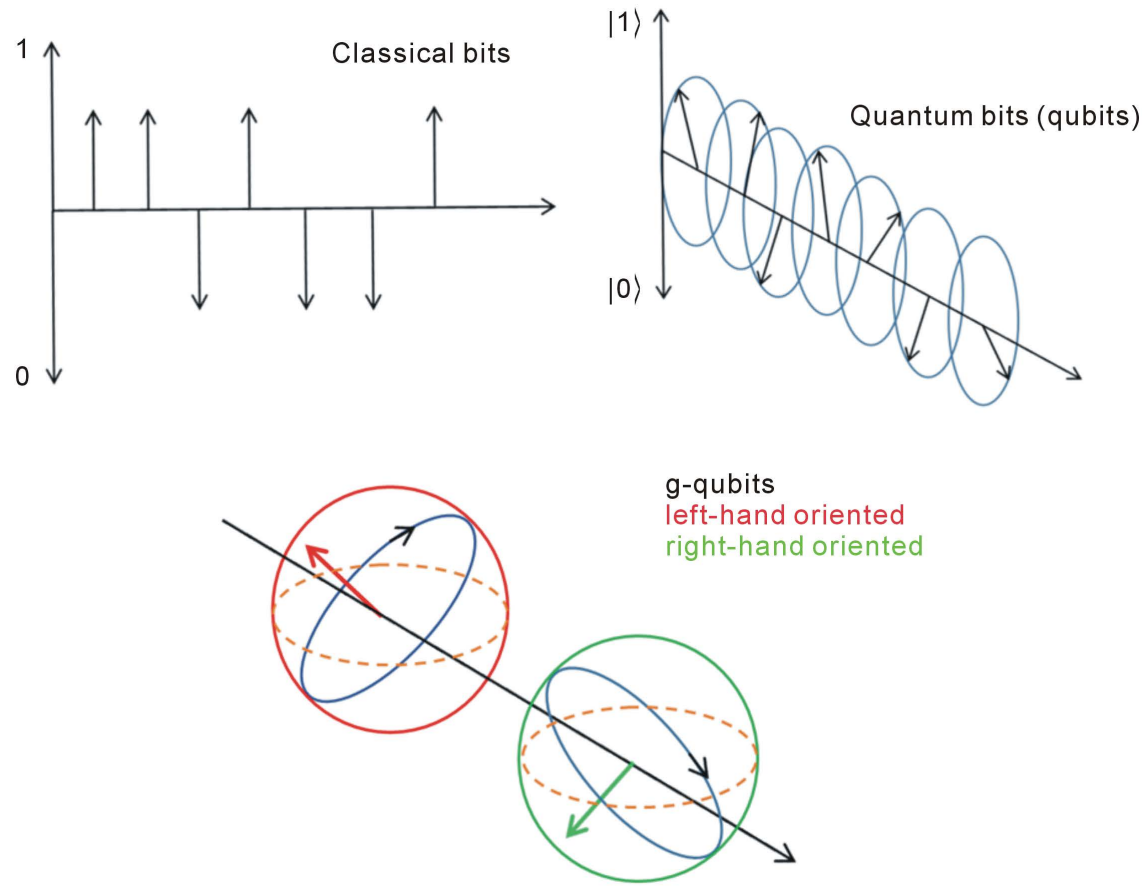

Figure 2. Differences between bits, qubits and g-qubits. 


$$
\left(\begin{array}{cc}
\gamma+\gamma_{1} & \gamma_{2}-i \gamma_{3} \\
\gamma_{2}+i \gamma_{3} & \gamma-\gamma_{1}
\end{array}\right)
$$

with removed not important scalar $\gamma$, has the lift in $G_{3}^{+}[3]$ :

$$
\mathcal{H}=I_{3}\left(\gamma_{1} B_{1}+\gamma_{2} B_{2}+\gamma_{3} B_{3}\right)
$$

Then the associated Clifford translation plane bivector is $-I_{3} \mathcal{H}(t)$. By normalizing the bivector to unit value we get generalization of imaginary unit

$$
i \Rightarrow I_{3} \frac{\mathcal{H}(t)}{|\mathcal{H}(t)|},
$$

that is critical for the whole approach. Therefore, for some $\Delta t$, Clifford translation for a given Hamiltonian is:

$$
\begin{aligned}
& |g(t+\Delta t)\rangle_{\left(\alpha(t+\Delta t), \beta(t+\Delta t), I_{S}(t+\Delta t)\right)} \\
& =\mathrm{e}^{\left.-\left(I_{3} \mid \mathcal{H}(t)\right)|\mathcal{H}(t)|\right)^{\mid \Delta t}}|g(t)\rangle_{\left(\alpha(t), \beta(t), I_{S}(t)\right)}
\end{aligned}
$$

For an arbitrary sequence of infinitesimal Clifford translations, the final state is integral ${ }^{2}$

$$
\int \mathrm{e}^{-I_{H(l)} \mathcal{H}(l) \mathrm{d} l}|g(l)\rangle_{\left(\alpha(l), \beta(l), I_{S}(l)\right)}
$$

along the curve on unit sphere $S^{3}$ composed of infinitesimal displacements by

$$
-\left(I_{3} \frac{\mathcal{H}(t)}{|\mathcal{H}(t)|}\right)|\mathcal{H}(l)| \mathrm{d} l
$$

Let's calculate the result of the right-hand side of (5) in general case when the plane of $I_{3} \frac{\mathcal{H}(t)}{|\mathcal{H}(t)|}$ differs from $S(t)$.

To calculate the geometric algebra product of the two exponents in Clifford translation with not coinciding exponent planes, $\mathrm{e}^{I_{S_{1}} \Delta \varphi_{1}} \mathrm{e}^{I_{S_{2}} \varphi_{2}}, S_{1} \neq S_{2}$, let's first expand $I_{S_{1}}$ in original basis to get formulas for generators of Clifford translation. If $I_{S_{1}}=\gamma_{1} B_{1}+\gamma_{2} B_{2}+\gamma_{3} B_{3}$ then a part of geometrical product $\mathrm{e}^{I_{S_{1}} \Delta \varphi_{1}} \mathrm{e}^{I_{S_{2}} \varphi_{2}}$ is:

$$
\begin{aligned}
& I_{S_{1}} I_{S_{2}} \\
& =\left(\gamma_{1} B_{1}+\gamma_{2} B_{2}+\gamma_{3} B_{3}\right)\left(\beta_{1} B_{1}+\beta_{2} B_{2}+\beta_{3} B_{3}\right) \\
& =-\left(\gamma_{1} \beta_{1}+\gamma_{2} \beta_{2}+\gamma_{3} \beta_{3}\right)+\left(\gamma_{3} \beta_{2}-\gamma_{2} \beta_{3}\right) B_{1}+\left(\gamma_{1} \beta_{3}-\gamma_{3} \beta_{1}\right) B_{2}+\left(\gamma_{2} \beta_{1}-\gamma_{1} \beta_{2}\right) B_{3} \\
& =-(\boldsymbol{\gamma} \cdot \boldsymbol{\beta})-I_{3}(\boldsymbol{\gamma} \times \boldsymbol{\beta})=I_{S_{1}} \cdot I_{S_{2}}+I_{S_{1}} \wedge I_{S_{2}}
\end{aligned}
$$

(see Figure 3)

where $\boldsymbol{\gamma}$ and $\boldsymbol{\beta}$ are vectors dual to bivectors $I_{S_{1}}$ and $I_{S_{2}}$.

Thus, the full product is:

\footnotetext{
${ }^{2}$ In the case of constant plane of Hamiltonian, it easily follows the Schrodinger equation of conventional quantum mechanics with clearly defined imaginary unit.
} 

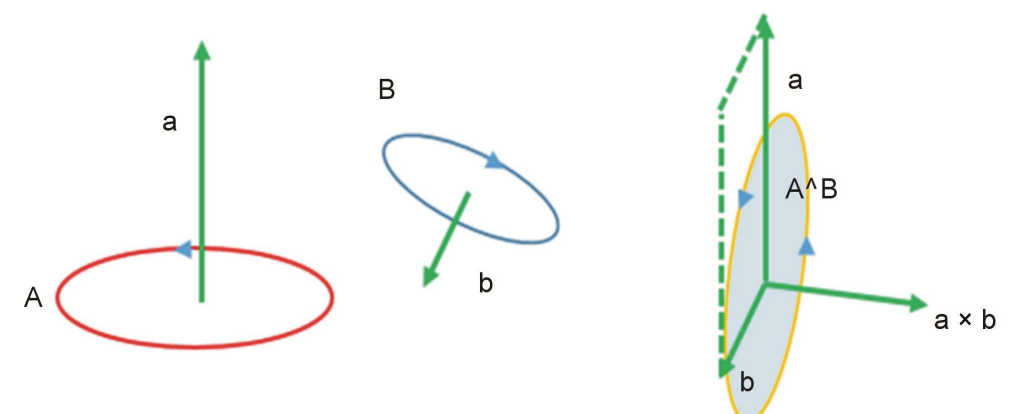

Figure 3. Two bivector geometrical product.

$$
\begin{aligned}
& \mathrm{e}^{I_{S_{1}} \Delta \varphi_{1}} \mathrm{e}^{I_{S_{2}} \varphi_{2}}=\cos \Delta \varphi_{1} \cos \varphi_{2}+\sin \Delta \varphi_{1} \cos \varphi_{2} I_{S_{1}} \\
&+\cos \Delta \varphi_{1} \sin \varphi_{2} I_{S_{2}}+\sin \Delta \varphi_{1} \sin \varphi_{2} I_{S_{1}} I_{S_{2}} \\
&= \cos \Delta \varphi_{1} \cos \varphi_{2}-\left(\sin \Delta \varphi_{1} \cos \varphi_{2} I_{S_{1}} I_{S_{2}}\right) I_{S_{2}} \\
&-\underbrace{-I_{S_{1}}\left(\cos \Delta \varphi_{1} \sin \varphi_{2} I_{S_{1}} I_{S_{2}}\right)+\sin \Delta \varphi_{1} \sin \varphi_{2} I_{S_{1}} I_{S_{2}}}_{\text {(scalar part) }} \\
&+\underbrace{\sin \Delta \varphi_{1} \cos \varphi_{2}+\sin \Delta \varphi_{1} \sin \varphi_{2}\left(I_{S_{1}} \cdot I_{S_{2}}\right)}_{\text {(bivector part, expansion in non-orthonormal basis } \left.I_{S_{1}}, I_{S_{2}}, I_{S_{1}} \wedge I_{S_{2}}\right)}
\end{aligned}
$$

\section{Transformations of Circular Polarized Electromagnetic Fields}

Now we have everything to retrieve action of Clifford translation generated by a Hamiltonian on general solution (4):

$$
\mathrm{e}^{-\left(I_{3} \mathcal{H}(t)\right)|\mathcal{H}(t)| \mathcal{H}(t) \mid \Delta t}\left(\boldsymbol{E}_{0}+I_{3} \boldsymbol{H}_{0}\right)\left(\lambda \mathrm{e}^{I_{S} \omega\left(t-\left(I_{3} I_{S}\right) \cdot \boldsymbol{r}\right)}+\mu \mathrm{e}^{I_{S} \omega\left(t+\left(I_{3} I_{S}\right) \cdot \boldsymbol{r}\right)}\right)
$$

To make expressions simpler I will use notations $I_{3} \frac{\mathcal{H}(t)}{|\mathcal{H}(t)|} \equiv I_{\mathcal{H}}$, $\omega\left(t-\left(I_{3} I_{S}\right) \cdot \boldsymbol{r}\right) \equiv \varphi_{+}$, and $\omega\left(t+\left(I_{3} I_{S}\right) \cdot \boldsymbol{r}\right) \equiv \varphi_{-}$. Then we get (see Sections 1.3 and 1.6 in [3] for multiplication details):

$$
\begin{aligned}
\mathrm{e}^{-I_{\mathcal{H}}|\mathcal{H}(t)| \Delta t}\left(\boldsymbol{E}_{0}+I_{3} \boldsymbol{H}_{0}\right)\left(\lambda \mathrm{e}^{I_{S} \varphi_{+}}+\mu \mathrm{e}^{I_{S} \varphi_{-}}\right) \\
=-\left(\boldsymbol{E}_{0}+I_{3} \boldsymbol{H}_{0}\right)\left(\lambda \mathrm{e}^{-I_{\mathcal{H}}|\mathcal{H}(t)| \Delta t} \mathrm{e}^{I_{S} \varphi_{+}}+\mu \mathrm{e}^{-I_{\mathcal{H}}|\mathcal{H}(t)| \Delta t} \mathrm{e}^{I_{S} \varphi_{-}}\right) \\
=-\left(\boldsymbol{E}_{0}+I_{3} \boldsymbol{H}_{0}\right) \lambda\left(\cos (|\mathcal{H}(t)| \Delta t) \cos \varphi_{+}-\sin (|\mathcal{H}(t)| \Delta t) \sin \varphi_{+}\left(I_{\mathcal{H}} \cdot I_{S}\right)\right) \\
-\left(\boldsymbol{E}_{0}+I_{3} \boldsymbol{H}_{0}\right) \lambda\left(\sin (|\mathcal{H}(t)| \Delta t) \cos \varphi_{+} I_{\mathcal{H}}+\cos (|\mathcal{H}(t)| \Delta t) \sin \varphi_{+} I_{S}\right. \\
\left.+\sin (|\mathcal{H}(t)| \Delta t) \sin \varphi_{+}\left(I_{\mathcal{H}} \wedge I_{S}\right)\right)-\left(\boldsymbol{E}_{0}+I_{3} \boldsymbol{H}_{0}\right) \mu\left(\sin (|\mathcal{H}(t)| \Delta t) \cos \varphi_{-} I_{\mathcal{H}}\right. \\
\left.+\cos (|\mathcal{H}(t)| \Delta t) \sin \varphi_{-} I_{S}+\sin (|\mathcal{H}(t)| \Delta t) \sin \varphi_{-}\left(I_{\mathcal{H}} \wedge I_{S}\right)\right)
\end{aligned}
$$

${ }^{3}$ In the case $I_{S_{1}}=I_{S_{2}}$ we trivially have rotation of $\mathrm{e}^{I_{S_{2} \varphi_{2}}}$ by angle $\Delta \varphi_{1}$. 
Let's take popular case of $I_{S}=B_{3}=\hat{x} \hat{y}$ (plane orthogonal to $\hat{z}$ axis) and $I_{\mathcal{H}}=B_{1}=\hat{y} \hat{z} \quad$ (or $I_{\mathcal{H}}=B_{2}=\hat{z} \hat{x}$, does not matter.) The above formula becomes:

$$
\begin{aligned}
& -\left(\boldsymbol{E}_{0}+I_{3} \boldsymbol{H}_{0}\right)\left[\lambda \left(\cos (|\mathcal{H}(t)| \Delta t) \cos \varphi_{+}+\sin (|\mathcal{H}(t)| \Delta t) \cos \varphi_{+} B_{1}\right.\right. \\
& \left.+\sin (|\mathcal{H}(t)| \Delta t) \sin \varphi_{+} B_{2}+\cos (|\mathcal{H}(t)| \Delta t) \sin \varphi_{+} B_{3}\right) \\
& +\mu\left(\cos (|\mathcal{H}(t)| \Delta t) \cos \varphi_{-}+\sin (|\mathcal{H}(t)| \Delta t) \cos \varphi_{-} B_{1}\right. \\
& \left.\left.+\sin (|\mathcal{H}(t)| \Delta t) \sin \varphi_{-} B_{2}+\cos (|\mathcal{H}(t)| \Delta t) \sin \varphi_{-} B_{3}\right)\right]
\end{aligned}
$$

It makes simpler if $F_{+}$and $F_{-}$are equally weighted, say both $\lambda$ and $\mu$ are equal to one:

$$
\begin{aligned}
- & \left(\boldsymbol{E}_{0}+I_{3} \boldsymbol{H}_{0}\right)\left[\cos (|\mathcal{H}(t)| \Delta t)\left(\cos \varphi_{+}+\cos \varphi_{-}\right)+\sin (|\mathcal{H}(t)| \Delta t)\left(\cos \varphi_{+}+\cos \varphi_{-}\right) B_{1}\right. \\
+ & \left.\sin (|\mathcal{H}(t)| \Delta t)\left(\sin \varphi_{+}+\sin \varphi_{-}\right) B_{2}+\cos (|\mathcal{H}(t)| \Delta t)\left(\sin \varphi_{+}+\sin \varphi_{-}\right) B_{3}\right] \\
= & -2\left(\boldsymbol{E}_{0}+I_{3} \boldsymbol{H}_{0}\right) \cos (\hat{z} \cdot \boldsymbol{r})\left[\cos (|\mathcal{H}(t)| \Delta t) \cos \omega t+\sin (|\mathcal{H}(t)| \Delta t) \cos \omega t B_{1}\right. \\
& \left.+\sin (|\mathcal{H}(t)| \Delta t) \sin \omega t B_{2}+\cos (|\mathcal{H}(t)| \Delta t) \sin \omega t B_{3}\right)
\end{aligned}
$$

\section{Action of Polarization States on Observables}

Since a state in the described formalism is operator that gives the result of measurement when acting on observable, which can be any element of geometric algebra $G_{3}$, the following is detailed description of the case when the element in parenthesis of the (6) expression acts on some bivector. Such operation is generalization of the Hopf fibration and rotates the bivector in three dimensions.

Denoting ${ }^{4}$ :

$$
\begin{aligned}
& \cos (|\mathcal{H}(t)| \Delta t) \cos \omega t+\sin (|\mathcal{H}(t)| \Delta t) \cos \omega t B_{1} \\
& +\sin (|\mathcal{H}(t)| \Delta t) \sin \omega t B_{2}+\cos (|\mathcal{H}(t)| \Delta t) \sin \omega t B_{3} \\
& \equiv \alpha+\beta_{1} B_{1}+\beta_{2} B_{2}+\beta_{3} B_{3} \equiv \mathrm{e}^{I_{\mathcal{H}, \omega} \omega^{\psi}}
\end{aligned}
$$

where

$$
\begin{gathered}
I_{\mathcal{H}, \omega}=\left(\gamma_{1} B_{1}+\gamma_{2} B_{2}+\gamma_{3} B_{3}\right) \\
\gamma_{1}=\frac{\sin (|\mathcal{H}(t)| \Delta t) \cos \omega t}{\sqrt{\sin ^{2}(|\mathcal{H}(t)| \Delta t)+\cos ^{2}(|\mathcal{H}(t)| \Delta t) \sin ^{2} \omega t}} \\
\gamma_{2}=\frac{\sin (|\mathcal{H}(t)| \Delta t) \sin \omega t}{\sqrt{\sin ^{2}(|\mathcal{H}(t)| \Delta t)+\cos ^{2}(|\mathcal{H}(t)| \Delta t) \sin ^{2} \omega t}} \\
\gamma_{3}=\frac{\cos (|\mathcal{H}(t)| \Delta t) \sin \omega t}{\sqrt{\sin ^{2}(|\mathcal{H}(t)| \Delta t)+\cos ^{2}(|\mathcal{H}(t)| \Delta t) \sin ^{2} \omega t}}
\end{gathered}
$$

${ }^{4}$ Easy to see that the left-hand side is unit value element of $G_{3}^{+}$. 


$$
\psi=\cos ^{-1}(\cos (|\mathcal{H}(t)| \Delta t) \cos \omega t)
$$

and taking a bivector operand (observable) $c_{1} B_{1}+c_{2} B_{2}+c_{3} B_{3}$ we get the result of measurement, action of the state on observable (see [3], [4] for details):

$$
\begin{aligned}
& \mathrm{e}^{-I_{\mathcal{H}, \omega /}}\left(c_{1} B_{1}+c_{2} B_{2}+c_{3} B_{3}\right) \mathrm{e}^{I_{\mathcal{H}, \omega /}} \\
&=\left(c_{1}\left[\left(\alpha^{2}+\beta_{1}^{2}\right)-\left(\beta_{2}^{2}+\beta_{3}^{2}\right)\right]+2 c_{2}\left(\beta_{1} \beta_{2}-\alpha \beta_{3}\right)+2 c_{3}\left(\beta_{1} \beta_{3}+\alpha \beta_{2}\right)\right) B_{1} \\
&+\left(2 c_{1}\left(\alpha \beta_{3}+\beta_{1} \beta_{2}\right)+c_{2}\left[\left(\alpha^{2}+\beta_{2}^{2}\right)-\left(\beta_{1}^{2}+\beta_{3}^{2}\right)\right]+2 c_{3}\left(\beta_{2} \beta_{3}-\alpha \beta_{1}\right)\right) B_{2} \\
&+\left(2 c_{1}\left(\beta_{1} \beta_{3}-\alpha \beta_{2}\right)+2 c_{2}\left(\beta_{2} \beta_{3}+\alpha \beta_{1}\right)+c_{3}\left[\left(\alpha^{2}+\beta_{3}^{2}\right)-\left(\beta_{1}^{2}+\beta_{2}^{2}\right)\right]\right) B_{3} \\
&=\left(c_{1} \cos 2 \omega t-\sin 2 \omega t\left(c_{2} \cos (2|\mathcal{H}(t)| \Delta t)-c_{3} \sin (2|\mathcal{H}(t)| \Delta t)\right)\right) B_{1} \\
&+\left(c_{1} \sin 2 \omega t+\cos 2 \omega t\left(c_{2} \cos (2|\mathcal{H}(t)| \Delta t)-c_{3} \sin (2|\mathcal{H}(t)| \Delta t)\right)\right) B_{2} \\
&+\left(c_{2} \sin (2|\mathcal{H}(t)| \Delta t)+c_{3} \cos (2|\mathcal{H}(t)| \Delta t)\right) B_{3}
\end{aligned}
$$

One interesting remark. If the observable belongs only to the $B_{1}$ plane, that's $C_{2}=C_{3}=0$, the result of measurement has only components in $B_{1}$ and $B_{2}$, projections of the value $c_{1}$ due to rotation with angular velocity $2 \omega$ around the $\hat{z}$ axis.

\section{Polarization States Acting on Multiple Observables}

The core of quantum computing should not be in entanglement as it understood in conventional quantum mechanics, which only formally follows from representation of the many particle states as tensor products of individual particle states and not supported by really operating physical devices. The core of quantum computing scheme should be in manipulation and transferring of sets of states as operators decomposed in geometrical algebra variant of qubits (g-qubits), or four-dimensional unit sphere points, if you prefer. Such operators can act on observables, particularly through measurements. From the recent calculation we realize that the action of state, which depends on $(t, r)$, on an observable can be done only if observable is defined at the same point $(t, \boldsymbol{r})$ where the state is defined [5]. In this way quantum computer is an analog computer keeping information in sets of objects with infinite number of degrees of freedom, contrary to the two value bits or two-dimensional Hilbert space elements, qubits.

Thus, if we have a state

$$
\alpha+I_{S} \beta \equiv|g\rangle_{\left(\alpha, \beta, I_{S}\right)}=|g\rangle_{\left(\alpha(t, r), \beta(t, r), I_{S}(t, r)\right)}
$$

as in the case of polarization defined states, it becomes a state acting on a set of observables if the latter are defined at some given points:

$$
\left|c_{n}\right\rangle=|c\rangle_{\left(c\left(t_{n}, r_{n}\right), I_{C}\left(t_{n}, r_{n}\right)\right)}=c_{0}\left(t_{n}, \boldsymbol{r}_{n}\right)+I_{C\left(t_{n}, \boldsymbol{r}_{n}\right)} \sqrt{1-c_{0}^{2}\left(t_{n}, \boldsymbol{r}_{n}\right)}, n=1, \cdots, N
$$

Then the state transforms into multi-observable one: 


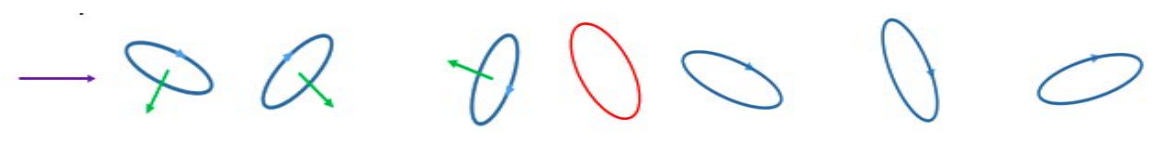

Figure 4. Decoding of g-qubit message.

$$
\begin{aligned}
& \left|g\left(\alpha\left(t_{1}, \boldsymbol{r}_{1}\right), \beta\left(t_{1}, \boldsymbol{r}_{1}\right), I_{S}\left(t_{1}, \boldsymbol{r}_{1}\right)\right) \cdots g\left(\alpha\left(t_{N}, \boldsymbol{r}_{N}\right), \beta\left(t_{N}, \boldsymbol{r}_{N}\right), I_{S}\left(t_{N}, \boldsymbol{r}_{N}\right)\right)\right\rangle \\
& \equiv\left|g_{1} \cdots g_{N}\right\rangle=\iint|g\rangle_{\left(\alpha(t, r), \beta(t, r), I_{S}(t, r)\right)} \delta\left(\boldsymbol{r}-\boldsymbol{r}_{1}\right) \delta\left(t-t_{1}\right) \mathrm{d} \boldsymbol{r} \mathrm{d} t+\cdots \\
& \quad+\iint|g\rangle_{\left(\alpha(t, \boldsymbol{r}), \beta(t, \boldsymbol{r}), I_{S}(t, \boldsymbol{r})\right)} \delta\left(\boldsymbol{r}-\boldsymbol{r}_{N}\right) \delta\left(t-t_{N}\right) \mathrm{d} \boldsymbol{r} \mathrm{d} t \\
& =\sum_{n=1}^{n=N} \iint|g\rangle_{\left(\alpha(t, r), \beta(t, \boldsymbol{r}), I_{S}(t, \boldsymbol{r})\right)} \delta\left(\boldsymbol{r}-\boldsymbol{r}_{n}\right) \delta\left(t-t_{n}\right) \mathrm{d} \boldsymbol{r} \mathrm{d} t
\end{aligned}
$$

This formula for $\left|g_{1} \cdots g_{N}\right\rangle$ bears clear physical and geometrical sense, contrary to conventional quantum mechanics definition following formally from tensor product which does not have good physical interpretation but is the root of entanglement-based quantum computing.

The formula also prompts how quantum encryption decoding can be effectively implemented with the bivector value security key (see Figure 4).

The formula can also be applied to challenging area of anyons in three dimensions.

\section{Conclusion}

Two seminal ideas-variable and explicitly defined complex plane in three dimensions, and the $G_{3}^{+}$states $^{5}$ as operators acting on observables-allow to put forth comprehensive and much more detailed formalism appropriate for quantum mechanics in general and particularly for quantum computing schemes. The approach may be thought about, for example, as a far going geometric algebra generalization of some proposals for quantum computing formulated in terms of light beam time bins, see [6], [7], but giving much more strength and flexibility in practical implementation.

\section{References}

[1] Soiguine, A. (2017) Quantum Computing with Geometric Algebra. Future Technologies Conference, 28-30 November 2017, Vancouver, Canada.

[2] Soiguine, A. (1996) Complex Conjugation-Relative to What? Clifford Algebras with Numeric and Symbolic Computations, Boston, Birkhauser, 284-294.

[3] Soiguine, A. (2015) Geometric Phase in Geometric Algebra Qubit Formalism. LAMBERT Academic Publishing, Saarbrucken.

[4] Soiguine, A. (2015) Geometric Algebra, Qubits, Geometric Evolution, and All That. http://arxiv.org/abs/1502.02169

[5] Soiguine, A. (2016) Anyons in Three Dimensions with Geometric Algebra. http://arxiv.org/abs/1607.03413

[6] Humphreys, P.C., et al. (2013) Linear Optical Quantum Computing in a Single Spa-

${ }^{5}$ Good to remember that "state" and "wave function" are (at least should be) synonyms in conventional quantum mechanics. 
tial Mode. Physical Review Letters, 111, 150501-1-150501-5.

https://doi.org/10.1103/PhysRevLett.111.150501

[7] Kok, P., et al. (2007) Linear Optical Quantum Computing with Photonic Qubits. Reviews of Modern Phys.ics 89, 135-174.

https://doi.org/10.1103/RevModPhys.79.135 